\title{
Myasthenia gravis: a patient's perspective
}

Mark Latham was the United Nations representative in the Cape Verde Islands. He was 56 years old when he developed myasthenia gravis in 1998.

\section{AP: What were the first symptoms you had?}

ML: I had some back pain due to all the packing and unpacking I do on my travels, but then I began to feel weaker. The orthopaedic opinion was that I had a disc problem and I was advised to rest in bed.

During this period of bed rest I noticed my arms were weak. It was difficult to hold a book to read. Over the next few weeks I experienced some difficulty chewing and swallowing.

AP: How was the diagnosis made?

ML: I had to travel to the UN headquarters in New York, but I was so weak I had to do the whole journey on a stretcher. When I got to the USA I was taken by ambulance to the emergency department of a large city hospital. My wife suggested that I had a neurological problem so a neurologist was called to see me. He quickly diagnosed myasthenia gravis and confirmed this by giving me an injection which promptly improved how I felt.

AP: How did you feel about the diagnosis?

ML: Myasthenia gravis did not mean anything to me, but I was glad that somebody now knew what was wrong with me.

AP: What treatment were you given?

ML: I was given prednisone and pyridostigmine. Within a week I was walking, but then I began to feel worse. I then collapsed. I was paralysed and only able to move my wrist and head. It was hard to breathe. I was admitted to intensive care and had three hours of plasmapheresis every other day for seven sessions. This had to be done through a femoral tap.

Azathioprine was added to my treatment and I gradually improved. Being able to sit up and turn myself over in bed were big achievements. While I was in New York I had my thymus removed.

AP: How were you able to get around?

ML: I lost a lot of muscle so I had to learn to walk again. When I was discharged from hospital I was in a wheelchair. I stayed in a hotel for a month and just had to practise walking up and down the corridor, first with a frame and then with a stick. I had physiotherapy three times a week. Going out of the hotel was difficult. I could not lift my feet over the smallest step and I fell over several times. When I went to hospital by taxi I was unable to get out of my seat.

\section{AP: Were you able to work?}

ML: When I returned to the Cape Verde Islands I waited a month to build up my strength before resuming work.

Back at work I found that I was easily fatigued. I could only sit in meetings for an hour. Having a chair with a high back helped to support my head.

AP: Have you had any relapses?

ML: About six months after returning to the Cape Verde Islands I started feeling increasingly weak particularly in the legs and neck. My eyelids started to droop and my breathing became laboured.

This time I was treated in Oxford, England. By the time I got there my eyelids were completely closed and breathing was very difficult. My eyes stayed shut for six weeks. The pyridostigmine and azathioprine were continued and the prednisone was increased. I also had plasmapheresis and I gradually recovered.

I retired to Sydney in 2000 and had one recurrence of weakness in 2002 when I experienced double vision. This was managed with plasmapheresis.

AP: Have you had any side effects from your treatment?

ML: The pyridostigmine gives me nausea and a headache. I had to have cataracts removed from both eyes as a result of the prednisone. I have also had a few skin cancers removed and now take mycophenolate in place of azathioprine.

AP: How are you managing now?

ML: My weakness can vary from day to day. On some days I can walk five kilometres, on others I can barely manage one kilometre. I am unable to run.

I am able to drive again, but manual transmission was a problem. In a traffic jam my left leg ran out of strength and I was unable to move the clutch pedal. I now drive an automatic.

The main problem now is fatiguability. I've retired, so the fatigue is manageable and does not interfere with my lifestyle. 